\title{
¿Es la artrosis de la rodilla, por fricción (plica), una realidad? Análisis de esta patología degenerativa articular. Informe de un caso
}

\author{
E. Nieto-Andueza ${ }^{1}$, J. R. Valenti-Nin ${ }^{2}$ \\ ${ }^{1}$ Profesor Titular de Ortopedia y Traumatología. Universidad de Los Andes (ULA). Mérida. Venezuela. \\ ${ }^{2}$ Profesor del Departamento de Ortopedia y Traumatología. Clínica Universidad de Navarra. Avenida Pio XII, 36. Código Postal. 31008.
}

\section{Resumen}

La osteoartrosis de rodilla es la causa más común de dolor en el adulto mayor, se trata de una afección secundaria a diferentes patologías y afecta todas las estructuras intraarticulares. Un posible factor etiológico podría ser secundario a una plica medial inflamada y engrosada. Esta modalidad de patología no ha sido considerada en ninguna de las clasificaciones existentes y puede ser diagnosticada previamente, en la mayoría de los casos, mediante una resonancia magnética y confirmada por artroscopia. Los autores actualizan el tema y presentan un caso de artrosis del compartimiento medial de la rodilla secundaria a una plica medial.

Palabras clave: plica medial; osteoartrosis de la rodilla; resonancia magnética; artroscopia.

\section{Abstract}

Osteoarthritis of the knee is the most common cause of pain in the elderly; it is a condition secondary to various diseases and affects all intra articular structures. A possible etiologic factor could be secondary to medial plica inflamed and thickened. This type of pathology was not considered in any of the existing classifications and may be previously diagnosed, in most cases, by an MRI and confirmed by arthroscopy. The authors present a case of osteoarthritis of the medial compartment of the knee secondary to a medial plica and the theme is updated.

Key words: medial plica; knee osteoarthritis; MRl; arthroscopy.

\section{Introducción}

La osteoartrosis de rodilla es afección frecuente en el adulto mayor y en ella participan de forma rutinaria la sinovial, el paquete adiposo infrapatelar de Hoffa, el hueso subcondral y el cartílago hialino que cubre el fémur, la tibia y la rótula.

En particular, la membrana sinovial (MS) es una estructura rica en células que producen un líquido viscoso, ligeramente alcalino con alta concentración de proteínas, glicoproteínas, proteoglicanos, fosfolípidos, hialuronato de glucosaminoglicanos (HA) y lubricina. Este líquido sinovial (LS) no es más que un ultrafiltrado de la sangre con aditivos con una serie de funciones biológicas como nutrir el cartílago, lubricarlo y proteger la superficie. Bajo presión, el HA contribuye a mantener las propiedades elásticas del cartílago mientras que la lubricina codificada por el gen PRG4, y sintetizada por fibroblastos similares a los sinoviocitos y los condrocitos superficiales, es una glicoproteína mucinosa que actúa de manera sinérgica con el HA para proporcionar: lubricación, disipar la energía, condroprotección y reducir el depósito de proteínas nocivas en la superficie articular ${ }^{1}$.

La MS elimina productos del metabolismo del cartílago y actúa como una membrana semipermeable que controla el tráfico molecular hacia y desde el espacio articular. El mantenimiento de la composición del LS es esencial para preservar el estado fisiológico normal del cartílago articular y en condiciones normales, las moléculas de alto peso molecular como la lubricina y HA no son fácilmente permeable, mientras que pequeñas moléculas como factores de crecimiento y citoquinas se difunden fácilmente a través de la $\mathrm{MS}^{2}$.

La rodilla se forma por un fenómeno de cavitación que

\section{Correspondencia}

Dr. E Nieto-Andueza, Urb. La Fontana. Apto. A 62.Av.Urdaneta. Mérida. Venezuela. Email: ejnieto@ula.ve Email Dr. J.R.Valenti-Nin: jrvalentin@unav.es 
se presenta alrededor de la séptima semana del desarrollo fetal; este proceso se ha estimado que podría ocurrir en tres tiempos sucesivos: primero fémoro patelar, después menisco femoral y finalmente menisco tibial, pero hasta el momento no hay consenso al respecto. Un remanente embriológico de este desarrollo es la plica medial y representa un pliegue de la membrana sinovial con una localización anatómica bastante constante porque va desde la pared medial de manera oblicua hacia abajo en el plano coronal, pasa por arriba del cóndilo medial y se inserta en la sinovia que rodea el paquete adiposo infrapatelar, entra en contacto con el cóndilo medial cuando la rodilla flexiona más de treinta grados $\left(30^{\circ}\right)$. Ha recibido otras denominaciones como: la cornisa, estante o repisa sinovial, plica sinovialis mediopatellaris, banda de lino, repisa de Aoki o elongata alaris ${ }^{3}$.

En relación a la plica medial, algunos autores estiman que durante el examen clínico y realizando algunas maniobras es factible diagnosticarla, pero la Resonancia Magnética puede ser la prueba que más orienta hacia la existencia de esta patología, incluso con un clasificación en tres variedades: A. Una lesión que consiste en una elevación similar a un cordón en la pared sinovial; B. Aspecto de estante o repisa, pero no llega a la tróclea medial; C. Gran apariencia de repisa y cubre la superficie anterior de la tróclea medial (Figura 1). La artroscopia confirma su existencia y gravedad de acuerdo con la conocida y simple clasificación de Sakakibara quien la divide en cuatro variedades: A. Similar a un cordón; B. Se comienza a insinuar como un pliegue; C. Un pliegue que cubre la superficie anterior y medial del cóndilo (Figura 2); D. Defecto en la parte central que da la impresión de doble inserción medial ${ }^{4,5}$.

El objetivo de esta presentación es llamar la atención sobre el papel de la plica sinovial medial, en la producción de artrosis del cóndilo sobre el cual desliza.

\section{Caso clínico}

Uno de los protocolos de investigación del departamento de Ortopedia y Traumatología de la Universidad de Navarra se refiere a la artrosis de rodilla en los pacientes de la tercera edad con sintomatología dolorosa e inflamatoria que no mejora por métodos habituales; a los cuales se les indica una resonancia magnética y una artroscopia si existen factores mecánicos que la justifique.

Presentamos un caso de artrosis por fricción bien documentado; se trata de un paciente de 75 años quien manifiesta dolor en la rodilla derecha, desde el mes de mayo del 2015, el cual aparece mientras caminaba sin

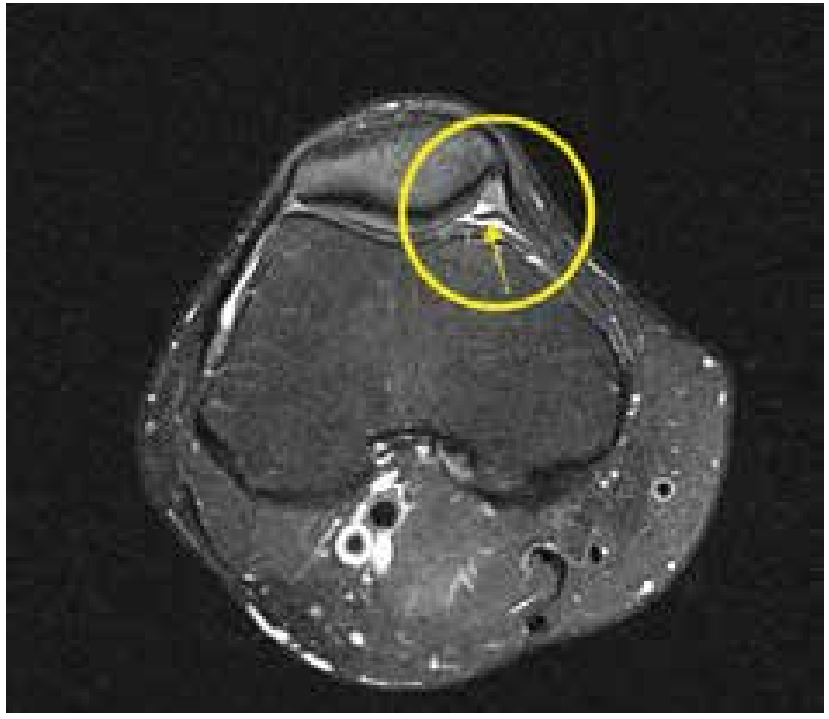

Figura 1. En el círculo se evidencia desde el punto de vista de la Resonancia Magnética una plica medial del tipo C4 (flecha).

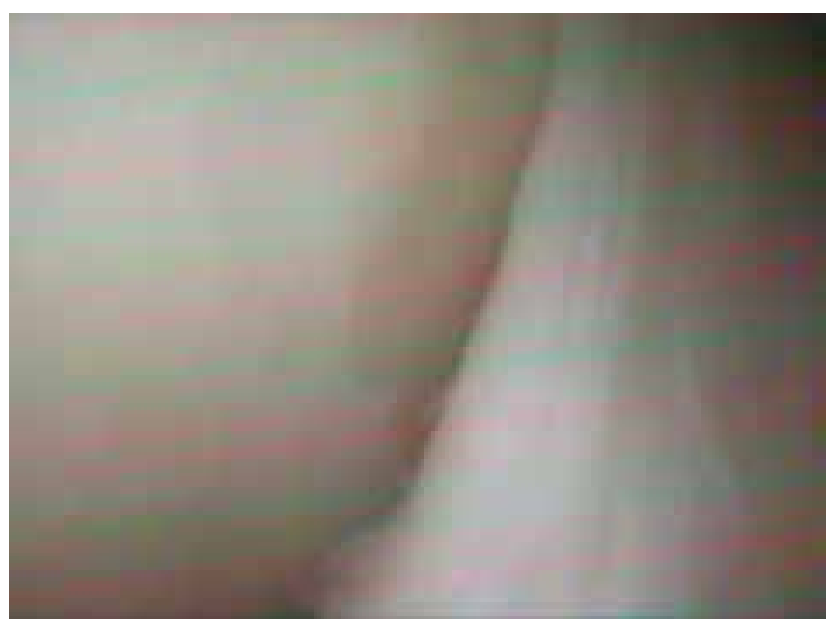

Figura 2. Plica medial tipo C de Sakakikabara ${ }^{5}$

haber realizado mal gesto asociado. Refiere aumento de volumen discreto. Entre los antecedentes de importancia una artroscopia previa en la rodilla izquierda. A la exploración física se encuentra un hidrops $(+/+++)$, cepillo negativo, no dolor en facetas pero sí en interlínea interna. Maniobras meniscales positivas para el menisco interno. Flexo-extensión $5^{\circ}-120^{\circ}$ con molestias en los últimos grados. La resonancia magnética evidencia rotura compleja del menisco interno asociada a lesión degenerativa del cóndilo medial grado IV. Se comentó que el menisco medial presentaba alteraciones morfológicas con aparente fragmentación, desplazados hacia el surco intercondileo, aunque no se trata de una "rotura en asa de cubo" (Figura 3). El día 16.6.15 se realiza artroscopia que pone en 
evidencia plica medial que se sobreponía a la artrosis del cóndilo medial y que los autores estimaron que pudiera ser la causante del desgaste (Figura 4).
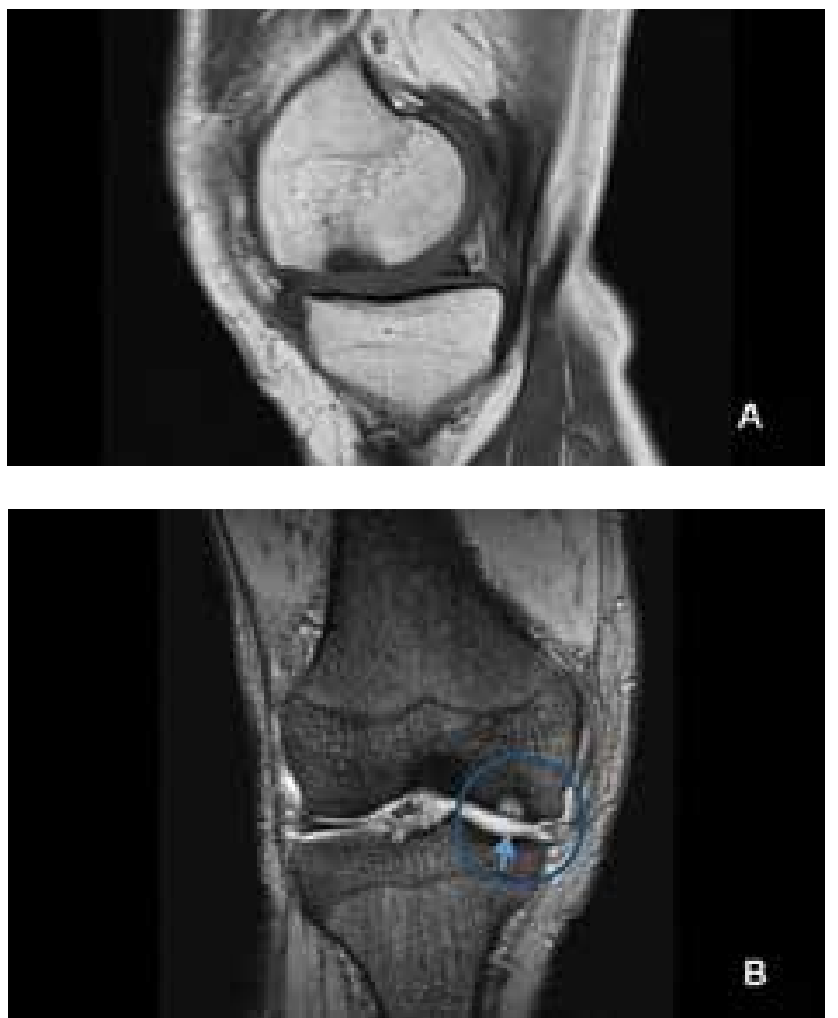

Figura 3. $\boldsymbol{A}$. En la resonancia magnética sagital se ve la importante lesión osteocondral en el cóndilo medial. B. En esta vista la flecha dentro del círculo se evidencia la lesión osteocondral e inmediatamente debajo una imagen que pudiera ser la plica medial.

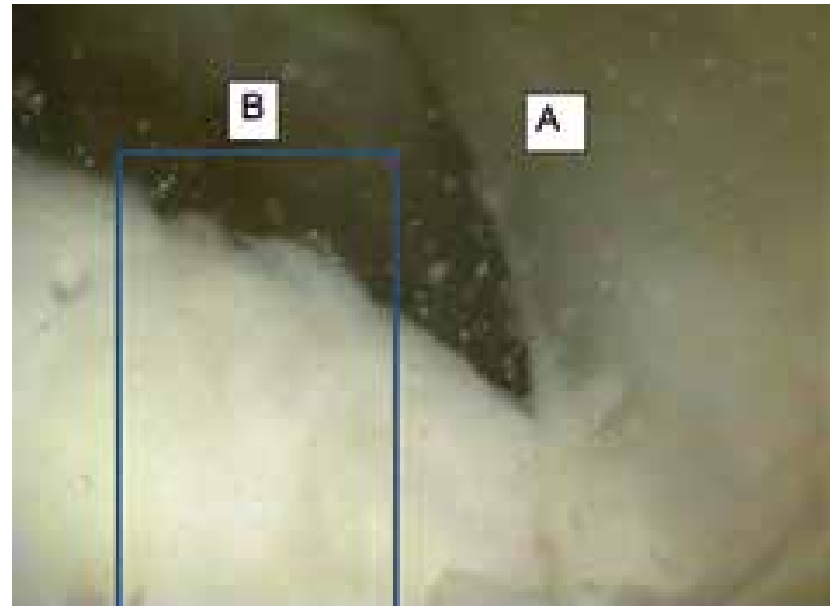

Figura 4. Detalle de la artroscopia: $A$. Plica medial del tipo $C$ de la clasificación de Sakakikabara ${ }^{5}$. B. En el rectángulo se ve el desgaste, casi lineal, que sigue todo el cóndilo femoral.

\section{Discusión}

La osteoartrosis $(\mathrm{OA})$ es el tipo más común de enfermedad inflamatoria articular, de inicio tardío, degenerativa, con pérdida del cartílago articular, se consideraba secundaria a sobrecarga, incongruencia articular o fragilidad del cartílago; hoy en día se sabe que es una enfermedad compleja multifactorial y multitisular. Ha sido clasificada como primaria o idiopática (¿genética?) o secundaria a sobrepeso, alteración de los ejes o traumas ${ }^{6}$.

La plica medial posee una prevalencia variable y de acuerdo a la literatura oscila entre un $19 \%$ y un $64 \%$, llegando a ser del 79,9\% en la población japonesa. En el caso particular de España, es del 9,1\%. De manera general, es sintomática entre el 3,8 y 5,5\%, su hallazgo pudiera ser significativo en rodillas con artrosis ${ }^{7-9}$.

Hoy en día, se está de acuerdo que la plica medial por microtraumas a repetición (sobre uso) se inflama, se engrosa, pierde su elasticidad y por esta razón es reconocida como una de las principales causas de dolor en el compartimiento anterior de la rodilla, de manera especial en los adolescentes y adultos jóvenes. Los síntomas más comunes incluyen dolor antero medial, aumento de volumen secundario a sinovitis, clic al caminar o seudobloqueo, debido a un fenómeno similar a una cuerda de arco sobre el cóndilo ${ }^{10}$.

La degeneración del cartílago, de manera frecuente, es secundaria a una variedad de trastornos internos de la rodilla, como los desgarros de ligamentos, rupturas meniscales, menisectomías y a las alteraciones de los ejes femoropatelares o femorotibiales.

La plica medial, como se muestra en el caso reportado (Figuras 3 y 4) a largo plazo puede conducir a una artrosis de rodilla y su gravedad se correlaciona de manera positiva con su severidad. Al reanalizar la Resonancia Magnética, lo que parecía fragmentación del menisco medial es factible que se tratara de la plica; esta modalidad de imagen no aparece en informes previos o en la clasificación ${ }^{4}$.

Durante las actividades diarias, la abrasión por la fricción repetida entre la plica medial y el cóndilo femoral medial irrita la sinovia e incrementa la producción de interleucina $1 \beta$ (IL-1 $\beta$ ), factor de necrosis tumoral alfa (TNF $\beta$ ) y metaloproteasas de la matriz 3 (MMP-3), lo que aumentaría la gravedad de la alteración patológica y daría lugar a síntomas clínicos como la sinovitis y el dolor asociado $^{11}$.

En revisión histórica se encontró que, en 1918, T Mayeda llevó a cabo una detallada evaluación anatómica de la plica medial y sugirió que podrían estar implicadas en las alteraciones intraarticulares. Con la llegada de la artroscopia, algunos estudiosos como E Vaubel, en los 
años treinta del siglo pasado, la comenzó a usar de forma rutinaria para el diagnóstico de afecciones articulares crónicas; él proporcionó el primer dibujo grabado de una imagen artroscópica que muestra una plica medial, pero lo más interesante es que está asociada a una enfermedad degenerativa localizada ${ }^{12}$.

Como se describe en párrafo anterior, desde hace muchos años se han realizado informes que relacionan la artrosis de la rodilla con la plica medial, pero ella tiene vigencia en tiempos recientes y de manera especial con los aportes de SR Lyu, quien la considera como productora de lo que él ha denominado la artrosis por fricción; en sus reportes encontró que el 66,9\% de casos de adultos mayores tenían esta patología y de ellos el $97 \%$ generaron destrucción del cartílago del cóndilo medial, por la combinación mecánica (fricción) y químicas por la elevada presencia de quimocinas producidas por el tejido sinovial ${ }^{13,14}$.

En resumen, el análisis de esta modalidad de patología nos permite concluir que la artrosis medial por fricción se debe tener en cuenta en pacientes de la tercera edad con patología inflamatoria de rodilla, en el momento de la valoración clínica y de la Resonancia Magnética. Porque la plica medial alterada lima poco a poco el cartílago hasta exponer el hueso subcondral; asociado a esto la sinovia produce citoquinas que incrementan el deterioro de la superficie articular. Lo que no está claro hasta el momento es el papel del tejido adiposo infrapatelar sitio de su inserción distal. A pesar de ello sigue siendo objeto de controversia, porque no está clara su naturaleza patológica y la necesidad de tratamiento, incluso algunos cirujanos sugieren que se pudiera estar sobreestimando o todo lo contrario ${ }^{12}$. Esta patología no ha sido considerada como factor etiológico de la artrosis en ninguna de las clasificaciones disponibles, por esta razón hay que tratar de detectarla de manera precoz y en este sentido la Resonancia Magnética parece ser de utilidad $^{15}$.

\section{Bibliografía}

1. Jay GD, Torres JR, Warman ML, Laderer MC, Breuer KS. The role of lubricin in the mechanical behavior of synovial fluid. Proc Natl Acad Sci U S A. 2007 Apr 10; 104(15):6194-9.

2. Scanzello CR, Goldring SR. The role of synovitis in osteoarthritis pathogenesis. Bone. 2012 Aug; 51(2):24957.

3. Ogata S, Uhthoff HK. The development of synovial plicae in human knee joints: an embryologic study. Arthroscopy 1990; 6(4):315-21.
4. Hayashi D, Xu L, Guermazi A, Kwoh CK, Hannon MJ, Jarraya M, et al. Prevalence of MRI detected mediopatellar plica in subjects with knee pain and the association with MRI-detected patellofemoral cartilage damage and bone marrow lesions: data from the Joints On Glucosamine study. BMC Musculoskelet Disord. 2013 Oct 12; 14:292.

5. Sakakibara J: Arthroscopic study on Iino's band (plica synovialis mediopatellaris). J Jpn Orthop Assoc 1976; 50:513-522.

6. Brandt KD, Dieppe P, Radin E.L. Etiopathogenesis of osteoarthritis. Rheum Dis Clin N Am. 2008; 34:531559.

7. Dupont JY. Synovial plicae of the knee. Controversies and review.Clin Sports Med. 1997 Jan; 16(1):87-122.

8. Romero GJ, Cabañas EL, F Jiménez DF, SánchezBrunete NP. Artroscopia de rodilla en adolescentes y preadolescentes. SECOT.Jun.2002. 46(3):246-253.

9. Nakayama A, Sugita T, Aizawa T, Takahashi A, Honma T. Incidence of medial plica in 3,889 knee joints in the Japanese population. Arthroscopy 2011; 27:1523-1527.

10. Christoforakis JJ, Sanchez-Ballester J, Hunt N, Thomas R, Strachan RK. Synovial shelves of the knee: association with chondral lesions. Knee Surg Sports Traumatol. Arthrosc. 2006 Dec; 14(12):1292-8.

11. Lyu SR, Chiang JK, Tseng CE. Medial plica in patients with knee osteoarthritis: a histomorphological study. Knee Surg Sports Traumatol Arthrosc 2010; 18:769776.

12. Schindler OS. 'The Sneaky Plica' revisited: morphology, pathophysiology and treatment of synovial plicae of the knee Knee Surgery, Sports Traumatology, Arthroscopy 2013; 22:2368.

13. Bellary SS, Lynch G, Housman B, Esmaeili E, Gielecki J, Tubbs RS, et al. Medial plica syndrome: a review of the literature. Clin Anat. 2012 May; 25(4):423-8.

14. Lyu SR, Lee CC, Hsu CC.Medial abrasion syndrome: a neglected cause of knee pain in middle and old age. Medicine (Baltimore). 2015 Apr; 94 (16):e736.

15. Singh R, Charles C, Skovrun ML, FitzGerald JD, Moreland LW, Kalunian KC. Validation of American College of Rheumatology classification criteria for knee osteoarthritis using arthroscopically defined cartilage damage scores. Semin Arthritis Rheum. 2005 Dec; 35(3):197-201. 